\title{
Seek, Share, or Withhold: Information Trading in MMORPGs
}

\author{
Authors: J. Tuomas Harviainen and Juho Hamari
}

\section{INTRODUCTION}

Information is one of the main types of vessels of value and target of economic transactions, coordination and production. With the advent of modern information and communication technologies (ICTs), information naturally has a more prominent and seemingly complex role in the economy. But what might be the most dramatic shift in the role of information in economic exchange is the intrinsic value of information goods, ranging from virtual items to digital music (Hamari, 2013; Lehdonvirta and Castronova, 2014). That is, information goods are increasingly the products that people pursue as the end products rather using them merely for coordinating other economics activities. In this article, we discuss the way in which information is such a valuable economic good in the context of online games (particularly MMORPGs or Massively Multiplayer Online Role-Playing Games - games that) and other persistent, virtual worlds. With virtual worlds, we (following Castronova, 2005, p. 11), mean expansive, world-like, large-group environments made by humans, for humans, and which are maintained, recorded, and rendered by computers. They are constructed, semi-separate realities that exist within what is commonly called the real world, stay active even when a player is not engaging with them, and can be contrasted with the real world.

MMORPGs are a type of online game that takes place in virtual worlds, with active player bases ranging from tens of thousands to millions. In them, players play fictional online personas, often referred to as 'avatars', which can interact with e.g., other players' avatars, system-controlled characters (called Non-Player Characters, or NPCs for short), the environment, and various types of monsters. The avatars gain virtual items, wealth, skills and powers as they develop. These can be acquired by completing game-internal tasks ('quests') and by defeating different types of foes - and by paying real-world money (Lehdonvirta and Castronova, 2014). Of particular interest are 'boss' monsters, powerful unique creatures that usually appear at key waypoints or ends of quests or specific adventure maps, require much group coordination and skill to defeat, and drop special items if the players' avatars triumph. Famous MMORPGs include Ultima Online (1997), EverQuest (1999), EVE Online (2003) and World of Warcraft (2004). The data for this article mostly comes from the last two, but research over the years has shown that the patterns discussed here also appear in many other MMORPGs (e.g., Castronova, 2005).

The worlds of MMORPGs and other virtual worlds, such as Second Life, are persistent, meaning that events keep taking place within them even while some players are currently not logged on to them. As this article will show, in the context of such environments information takes many forms, ranging from gameworld-internal parts of storylines, quests and item properties, to systemic (functions of algorithms within the game; the fact that the virtual items consist of information), to meta-game information exchanged during or outside of play by the players. For the purposes of this article, we therefore define information as "the potential message content in any piece of data, ranging from verbal statements to physical objects. It is selectively ignored or appropriated into knowledge structures by persons seeking or encountering it" (Harviainen, 2012, building upon Ingwersen and Järvelin, 2005).

The study of information, information goods, information trading and information practice trading in virtual environments is as important as in any other context, since virtual worlds are inevitably economies (Castronova, 2005) in a similar manner as any other organization of economics actors who conduct trade and make decision about labor, distribution of wealth and how they allocate their resources. The system designers, and often also players, exert power through economical processes, whether those processes are designed or emergent, overt or hidden (Lehdonvirta and Castronova, 2014). Because of this, understanding human behavior in such worlds without understanding the economics of the system is very difficult. Moreover, these 'virtual' environments provide a rich context for observing such information economic processes that also carry back into the 'real' world.

Based on theories of emergent social processes of information systems, our research question can be articulated as "how do social information practices function as means of trade in MMORPGs?" We combine material from several frameworks; particularly from library and information systems theories that also encompass management information systems and information economies (e.g., Buckland, 1991), views on cognitive approaches to management information systems (e.g., Land, 1992), and the study of virtual economies (e.g., Castronova, 2005; Hamari and Lehdonvirta, 2010; Lehdonvirta and Castronova, 2014; Malone, 2009). This is because we, following Buckland (1991), believe that the social properties of information systems become sufficiently visible only when their uses are analyzed from multiple perspectives. As noted by Vakkari (1999), building upon Checkland and Holwell (1998), retrieval systems must be understood in the context of information seeking, as they are actually combinations of two systems: the one being served (people), and the 
system that does the serving (the IR itself). In this article, we go one step further: we believe that in virtual worlds, the chain continues from retrieval to not only information seeking, but also other information practices.

Virtual worlds too consist of two information systems: one technological, the other a social system. The first of those is the "game proper", its code and partly the technology on which it functions. The technology and its code define the borders, the laws and the restrictions of the economy; how economic actors can act in the environment which correspond to the physical laws of the 'real world' (Lastowka and Hunter 2004; Castronova 2005; Lehdonvirta and Castronova, 2014). The second one arises from the social interplay of the players (Harviainen and Savolainen, 2014; see also Stenros, Paavilainen and Mäyrä, 2011). Buckland (1991) divides information systems into two key types, and MMORPGs exemplify that division: in them, the code level is an information-supplying system that retrieves information, documents and virtual objects, while the social level is also a system that informs, accessed cognitively (see Shaw and Culkin, 1987). Players navigate these two systems, paying for group membership and access to content with not only money and time, but also through information practices: socially, economically and culturally established ways in which they identify, produce, share, seek, use and even withhold information (as per Savolainen, 2008; see also Hamari and Lehdonvirta, 2010; Hamari, 2011). They furthermore do so according to personal and communal needs and expectations. It has earlier been discussed how some players function as information brokers or use limited local information for arbitrage (e.g., know where to buy some item cheap and where to sell it at a higher price; Lehdonvirta and Castronova, 2014, p. 8), how limited access to information affects prices and reliability of trade in virtual worlds (ibid, pp. 130-131), and optimize their performance (Paavilainen et al., 2013, p. 802), but we argue that information practices actually play a much larger role.

We take a meta-theoretical approach, building upon earlier key studies, such as Jørgensen's (2013) research on games as information ecologies, Harviainen, Gough and Sköld's (2012) work on game-related information phenomena, and Harviainen and Savolainen's (2014) analysis of synthetic worlds as information systems, as well as a survey of existing research. By drawing upon existing empirical works and synthesizing their results, we gain access to emergent, new data (Galliers, 1992, p. 158). This allows us to address the practices on a systemic level, drawing upon earlier findings that are placed within a wider context.

To understand how information practices become commodified in virtual worlds, it is necessary to analyze the context in which that commodification takes place. Virtual worlds are so exceedingly complex information systems that any such analysis is inevitably focused on just some particulars (Sköld et al., in press). In this article, we have therefore focused on prominent factors that in our view have the most direct impact on the information trade of MMORPGs and which are strongly emergent umbrella phenomena. We first turn our attention to the core of how the game functions as a set of information systems and how it related to the accumulation of information as social capital. Next, we discuss intra-game groups such as guilds as information organizations, as well as how they influence members' information practices. Then, we analyze the ways in which real money injected into the game economy (commonly referred to as Real Money Trade - RMT) changes the rules of this process, allowing access to additional content, new information and the bypassing of certain typical requirements. Finally, we show how the risk of information overload governs these processes, making the non-sharing of some information also a transaction.

\section{THE MMORPG AS AN INFORMATION SYSTEM}

As proposed by Harviainen and Savolainen (2014) and Sköld et al. (in press), virtual worlds consist of two (or more) information systems. At their core is the coded system, which functions like an extremely complex information retrieval (IR) system, the interface of which is an integral part of that system (Jørgensen, 2013). The same way as a management information system or a library database, the MMORPG's coded core is a set of elements that players can, through interaction (play), draw into contact with them. One key difference, however exists: whereas managerial information systems and their ilk are designed for ease of use, games are by intent made challenging, engaging and intrinsically motivating (Hamari, 2013; Hamari et al., 2014; Huotari and Hamari, 2012; Lehdonvirta and Castronova, 2014; Koivisto and Hamari 2014). That is a key part of their attraction, and playing is a skilled activity that often needs finesse.

Quests that are given in the game by non-player characters are in essence retrieval tasks, some of which are more explicit than others: for example, reconnaissance of a location clearly counts as an information search, and certain quests only become available (or areas meaningful to play), once the character has access to specific information within the game (e.g., only after a non-player character has in EverQuest or World of Warcraft told the player's avatar to do something specific in an area, such as "kill the Kobolds hiding in the ruins", the Kobolds can be found at that location - or they are there, but will not drop a certain item when killed). In information studies' terms, affordances within the game function as its content retrieval "keywords", the use of which provides cognitively, situationally and/or motivationally relevant results (as per Sarasevic, 1996; Cosijn and Ingwersen, 2000). As all enemies also consist of code (i.e., are algorithmically relevant retrieval results; ibid.), even fighting monsters is, from a systemic perspective, information retrieval (Harviainen and Savolainen, 2014). Together, the graphic representations, the challenge, the narrative, the rewards and so forth, give the code an experiential meaning, so that the retrieval tasks feel like the activities that they represent (see Hamari and Eranti, 2011). In other words, the interface makes the tasks seem something more than just intelligible strings of code or a dull information retrieval task in an office. The 
interface may attempt to immerse the player into a world beyond the dull reality by invoking curiosity, imagination or by simply placing the person into a specific and explicit fiction world. The player may know that the boss dragon is just an algorithm, but fighting it will first and foremost feel like fighting a virtual creature (Jørgensen, 2013). At the same time, players will nevertheless also be studying the code through the creature's actions, in the hopes of seeing hints on how to defeat it more efficiently. The study and its results include highly varying practices, ranging from improved team tactics to players reducing their computers' graphic levels so as to bring the whole battle as close as possible to just opposing an algorithm.

Comprehending how this underlying system functions is necessary for understanding the value of information in virtual environments, as the trade in information practices only makes sense in relation to player access to the IR system. While the social game world provides the context that influences the ways in which players access the IR system (as per Cosijn and Ingwersen, 200, p. 535), access to retrievable content determines what they can do during play.

Players experience the code first and foremost as play and content, much of it in the form of tasks. High-difficulty tasks such as defeating boss monsters require training to complete, and that training, in turn, requires new information, which needs to be sought before it can be applied for learning. In the IR system, information is everywhere, but only accessed through relevant key points, and often present as internal metadata. For example, the described properties of each in-game item are additional documentation on a game-internal document (the virtual item itself, such as a magical sword). In learning to fight a dragon-like boss, players study ways in which to respond to its algorithm (Vesa, 2013). They do so by not just practice (which, in information studies terms, is a kind of repetitive, collaborative retrieval from the IR core; see e.g., Hansen and Järvelin, 2005), but also the reading of online sources (such as discussion forums) and watching videos (e.g., Chen, 2009). What they use as information sources is material created by other player, i.e., the results of information creation and/or sharing, another central facet of information practices (see Savolainen, 2008). In essence, player are reverse-engineering information systems while playing the game, by using information and know-how acquired from multiple sources that are not restricted to those sources in the actual game. In essence, players are attempting to master an information system that is intentionally difficult to use, and are having fun while they are at it (Paavilainen et al., 2013; Hamari, 2015).

These practices are parts of the second systemic layer, which consists of one or more social information systems, all built upon the IR system ground. Land (1992) states that an information system is a social system, in which the information technological parts are embedded. Following Harviainen and Savolainen (2014) we, however, see the social system(s) as constructed on top of the IR artifact. Whereas a management information system, as described by Land (1992), can be perceived as a technical extension of other social practices, the social systems of MMORPGs would not exist without the games themselves. The social systems of MMORPGs consist of player conventions, habits, discourses, as well as information practices, and all of these tie together into the wider set of what can be described as interconnected participant practices. In that system, players who have access to more information have access to potentially more game content, and as a result the ability to share that access with others. This is highly important, as user attention is a scarce good likely to wane if not properly supported, and new content is a key tool for that purpose. As a result, content too is a scarce good, losing value the sooner, the frequently and the more it can be used or explored. For this reason developers intentionally control the revealing of information/content by controlling the skill progress of avatars, difficulty of tasks and so forth (Hamari and Lehdonvirta, 2010; Lehdonvirta and Castronova, 2014, pp. 9-20).

Because of the artificial scarcity, information becomes capital, as well as something that can be given, sold and bartered. Those who have more are in a position of strength, able to aid others in their tasks, grant access to some additional content (by e.g., telling how a puzzle is solved or a boss' weakness) - and to possibly spoil game experiences by telling too much. To engage in information sharing is to use power and to barter (Harviainen and Savolainen, in 2014). Information practices are traded within the game's extended system practically all players frequently use them to pay for goods and services, particularly in intra-community situations. More precisely, practices such as creating, seeking, sharing and withholding information function as traded services, while the information itself is a good. For example, one may pay for help in a task by telling the helping player a piece of valuable information, earn some additional loot on a raid by obtaining tactical information for the group in advance, or garner good will by sharing a helpful detail without any price (thus being possibly paid in reputation instead). This reputation-gaining also motivates people to post game walkthroughs and videos online, with free access for other players.

As pointed out by Castronova (2005), persistent virtual worlds are inevitably economies since people partake in economic transactions and decisions in them. As soon as people want things from each other, and a means of exchange exists, a market will emerge, by itself if not by intentional design (Lehdonvirta and Castronova, 2014, p. 57), given that mechanisms exist that make the trade possible. This is also true of the information market, as while information may in theory be available to all, not everyone has access to the right tidbits at the time when they are needed. What makes the information part of the game economy peculiar, however, is the way in which information can be traded without losing it. Whereas other types of goods or potential currencies tend to get diminished from one party when they are shared or traded (e.g., gold is given away), information sharing does not normally remove anything from the sharer (Lehdonvirta, 2009). Information is usually nonrivalrous and nonexclusive, meaning that it can be shared without losing it and that it is in theory available for 
everyone. Publishers and other authorities can, however, drastically reduce those traits, through processes of artificial exclusivity, but they cannot control all information in a system in that manner (Hamari and Lehdonvirta; 2010; Lehdonvirta and Castronova, 2014, p. 42). This leads to information trading between players.

The information trade may, as will be shown below, reduce the value of the content to which it gives access, as its novelty may wear off (Shapiro and Varian, 1999). In this, information goods are not different from any other goods that can rely on novelty for their value. Therefore, trading in information carries its own risks, as well as the chance that if one shares too much, the garnered capital will be lost. On the other hand, free sharing may sometimes create social capital of another kind, in the form of goodwill (see, e.g., Bourdieu, 1984). As in the real world, ownership of also virtual goods and information tends to be about the presentation of one's status (Hamari and Koivisto, 2013; Lehdonvirta, Wilska and Johnson, 2009). To be in the position of being able to share or trade information is thus power, and to be known as someone in such a position translates into status (Harviainen and Savolainen, 2014). To describe these processes, Consalvo (2007, pp. 184-185) uses the concept of 'gaming capital', which reflects the skills, status and information needed to become a respected member of a game-related subculture.

Land (1992) states that most information systems work with three simultaneously existing (sets of) information sources: the real world, which (as pointed out by Klabbers, 2009) acts as a key referent even in more fantastic settings, the designed information system (artifact) itself, and an informal information system constituted of and by the players. The latter two of these correspond with the IR system and social system described in this article, whereas the first, the real world as a referent, provides the additional information that is required to fluently use those two systems. In play, players use all three as information sources.

Therefore, the larger, social system of the game does not function without information brought into it by players (Crookall, Oxford and Saunders, 1987). Likewise, IR cores alone do not function without external information, either (see Ingwersen and Järvelin, 2005). The game-as-artifact itself is insufficient for play (Klabbers, 2009). This is because games as play-processes rely on existing real world concepts, such as understanding the basics of what "combat", "money" or "on the same side" may mean. This fact, combined by the various things people search for in games and the way catering for such interests affects game structure (see, e.g., Bartle, 2014), gives rise to highly varying play practices. Organizations develop to cater for such shared interests. Guilds, corporations and similar groups exemplify this in MMORPGs, which is why we now turn to analyze their relationship with the games' information economies.

\section{GUILDS, CORPORATIONS, CLANS AS INFORMATION ORGANIZATIONS}

Many players prefer to play together with others (Kallio, Mäyrä and Kaipainen,2011). MMORPGs further foster playing together, by having some content that is only accessible in large groups (Williams et al., 2006). This is in line with the fact that most users of information systems operate as parts of groups, especially in work contexts (Land, 1992), especially when considering that many game organizations take their tasks with work-like seriousness (Vesa, 2013; Warmelink, 2014). Exemplifying these are World of Warcraft raid guilds, which focus on high-end content that requires coordinated multiplayer activity in order to defeat powerful enemies (see Vesa, 2013; Williams et al., 2006). Defeating the boss-type enemies on raids may require hours upon hours of coordinated practice, as well as gathered information on how to accomplish the task (Chen, 2009). Participants contribute to the guild several things of value: their time, skills, things of in-game value (such as gold or powerful items) - and information in a same manner as employees exchange their time and skill for wages.

Because organized tasks such as World of Warcraft raids or EVE online production are collective activities, their related learning processes, too, take place communally. In this, guilds and EVE corporations exemplify Chirag Shah's (2012) model of collaborative information seeking in all of its five facets: collaboration (working together synergistically to achieve a common goal), cooperation (agents following some roles of interaction), coordination (connecting different agents in a harmonious action), contribution (offering of an individual agent to others) and communication (exchanging information between the agents). They also embody the ambiguity and group tension involved in such practices (see Vesa 2013; Warmelink, 2014; Shah 2012). In order to be considered active and contributing members of a guild, players have to take part of this shared task. In some groups, the leaders can even keep track through information and communication technologies (ICT) of which of the members contribute sufficiently. Specialized systems have been designed for the purpose of tracking the contributions and the 'wages' of players in guilds (how many items of value the player has received from the guild). These systems are commonly referred to as DKP (Dragon Kill Points) systems (see e.g. Malone, 2009; Silverman and Simon, 2009; Chen, 2009). Active groups tend to utilize a wide array of ICTs, which assists in both the intra-group sharing and the tracking of contributions (Warmelink, 2014, pp. 106-109).

Guilds differ from many similar-seeming real world organizations. This is due to a combination of the voluntary nature of play, the more playful structures of MMORPG organizations (especially those including easy entrance and exit; see Warmelink, 2014) and the high importance of information seeking for functional play. Whereas the ICT systems of hierarchical corporations tend to provide aggregated information that leads to an illusion of knowledge on the managerial level (see e.g., Mintzberg, 1983, pp. 243-248), MMORPG 
organizations require both highly accurate information in order to accomplish their goals (such as defeating a specific boss) and enough flexibility to keep their members motivated. The information required by players to e.g., defeat bosses or coordinate assaults against rival EVE corporations is also likely to be very detailed, whereas large organization such as real-world corporations, operating on a strategic level and in a very complex environment, have to deal with massive quantities of information, much of which is thus by necessity less exact. While game environments may well contain so much information that exactitude becomes impossible, in them (unlike in the real world) it tends to be possible to isolate a situation so that players are able to focus on only task-relevant information. The result of this is that collaborative information seeking is for MMORPG organizations a much more efficient method than managerially guided searches would be (something that sets many guilds in this regard apart from, e.g., soccer teams, which can also be playful and task-information dependent, yet have a tighter manager/coach/player role division).

On the other hand, due to the seeking-motivating voluntary nature of the play, participants may also be less likely to provide as much information to those in positions of power as they would in a work organization. Effectively, most of what is sought is utilized on the tactical, not strategic level, and while members have more leeway to seek information, they also have less duty to funnel it to those above. Thus, they tend to opt for just general-level sharing, as a matter of convenience. On the other hand, they have less incentive to manipulate or hide information from their colleagues in order to get an advantage than real-world corporate workers do (see e.g., Land, 1987). Therefore, while the guild structure may excel at the game-tasks it has at hand, a more traditional information structure (including the ICTs supporting it) is still more efficient for real-world purposes, and one that members may also prefer because it grants them personal advantages (Warmelink, 2014). Furthermore, as pointed out by Vesa (2013), organization members may be loyal to the organization, their own circle of associates within it, or just their own goals, and the lack of tight control systems (e.g., contract, salary) accentuates the organizational dissonance this causes.

As noted by Warmelink (2014, p. 107), ICT deployment in intra-game organizations may be conducted with the interests of the organization in mind, but in a manner designed to strengthen expertise and trust hierarchies, negotiated labor division and non-authoritative leadership, instead of following and supporting a formalized hierarchy. Instead of an emphasis of the current structure of power, they become information systems of constantly negotiating that power - systems of an information trade.

Buckland (1991) divides facets of information systems into three types, based on purpose: 1) A cognitive system leads to the user becoming informed, 2) political and managerial systems, in turn, function as information-supplying systems, and are tools for resource and information allocation. Finally, 3) economical systems define whether the use of an information system is seen as a worthwhile task. These facets can, and usually do, overlap. Games like World of Warcraft even support such overlap, by providing customizable player interfaces and different character types that crucially support each other during challenging play such as fighting a boss. Whereas the information system of a MMORPG combines the cognitive system of enjoyable play with the economic system of making the play easy, fluent and rewarding enough, guilds and corporations have to also utilize the managerial and political system, in order to keep functioning. Access to new information, including more play content, provides the additional economic system parts to offset the bureaucratic and hierarchical things that necessity adds to the ICT use - and the game experience itself. This is a type of information trade of its own: because guilds can't rely on contracts, an agreement to more managerial-centered approaches to information is rewarded with access to more information.

Guild and corporation members furthermore engage in what McKenzie (2003) calls "information seeking by proxy" - provision of unsolicited information from a peer. Non-guild players also engage in such practices, but often in a surreptitious manner. This is because being seeing as not knowing certain things carries with the stigma of inexperience ("n00b") status, exemplified by questions like "how u mine 4 fish?" that show the person asking has not devoted time to even the most basic of research or experimentation. A person's representative avatar (including texture, gear, etc.) furthermore easily defines whether that person is considered potentially in the know. As a result, even information professionals within virtual worlds have to hone their appearances, in order to be seen as credible (Mon, 2012). Those who have information hold status, capital and the ability to wield power over those who lack that information. This not only facilitates information trade, but also guides many potential purchasers of information to seek out shared, out-of-game information (e.g. forum posts, videos) instead (Harviainen, Gough and Sköld, 2012). The information is oftentimes not traded, because to engage in the purchase would label the one purchasing it (whether with e.g., gold or just the attributed social capital of a "thank you") inexperienced. Sometimes, however, players use other means of bypassing the issue, means to which we turn next.

\section{REAL MONEY TRADE AS INCREASED INFORMATION ACCESS}

The basic conventions described above start to break down, when real money enters play or some vessel of value is brought into the game that has been earned outside the system. Through systems such as micropayments, players can gain access to additional game content, better items, and so forth. This includes the ability to dig up and potentially share information on areas of the systems to which not everyone has access without RMT. Players use such information sharing, especially if they can do it faster or better than others, as a source of social capital. 
RMT has traditionally referred to the trade of virtual items, currency and information in the secondary market between players. Game publishers commonly frowned upon such activities and had several differing strategies to prevent it (Lehdonvirta 2005; Hamari and Lehdonvirta 2010). However, in the long run these efforts were deemed ineffective as well as they restricted player interaction further. Moreover, the game developers were missing out on the economic value of those trades between players. Therefore, game developers started to developed strategies to facilitate RMT in a couple ways: 1) By selling items themselves (spawned the Free-to-Play revenue model), 2) Facilitate player-to-player trade and charging from transactions (e.g. Steam).

An ironic aspect of RMT is the fact that games can be considered as the pinnacle of self-purposeful information systems (Hamari, 2015). This implies that the process of using these systems is presumably intrinsically motivating and valuable in itself. This notion would predict that people desire to play these games as much as possible (or in the context of argumentation in this article: players would desire to participate in information seeking activities first-hand). However, with RMT, players purchase information services that enable them to play games less. This further implies that some of the information practices outlined herein do not fulfill the description of intrinsically motivated activities and further imply that some of these activities are motivated by some other factors than the sheer enjoyment. Slightly exaggeratingly it can be said that people are more willing to pay money for game-related information and items than partake in-game activities that would eventually lead into the same outcome. Moreover and as described above, the value of many in-game items is defined in terms of their rarity. Therefore, as a result of RMT, the information value of certain things in play (e.g., owning a powerful item that shows the player's level of commitment to others) also radically diminishes (Lehdonvirta and Castronova, 2014, p. 145).

These notions in mind, game publishers have started to design games in a way that further create demand for such information services by creating artificial obstacles as well as limit player information on various aspects of the game thus effectively making the game more difficult and sometimes burdensome (Hamari and Lehdonvirta, 2010; Hamari, 2011; Hamari and Järvinen, 2011; Paavilainen et al., 2013; Alha et al., 2014). They also seek to commodify gaming capital, sometimes even selling back to players the paratextual information created by other players about a game, in the form of e.g., RMT-purchased guides (Consalvo, 2007).

While from a social perspective this purchasing of information and information goods can be seen as an unfair advantage, or even cheating (Alha et al., 2014; Lin and Sun, 2011; Paavilainen et al., 2013) it is nevertheless an intended part of the system. Many publishers actually gather most of their revenue precisely through such monetization processes, so the game as a system may have been designed for the very purpose of supporting and promoting RMT (Hamari and Lehdonvirta, 2010). As a result, the information trade of MMORPGs is splintering. Whereas on one hand, organizations such as guilds use information seeking and sharing as measures of member dedication, other players may value the freedom gained by access to shortcuts (see Consalvo, 2007). They may furthermore thus favor very different ways of paying for the game experiences with real money - whereas the one committing time and effort favors subscription fees, the shortcut taker is likely to favor micropayments that give access to extra content (see e.g., Lehdonvirta and Castronova, 2014).

\section{OVERLOAD AND SOCIAL NEGOTIATION}

Having too little information prevents the meaningful use of the game. Having too much information (e.g., spoilers), likewise, may make the game uninteresting or even unplayable since it may become too easy or lacking in sufficient novelty (Consalvo, 2007). This is a type of information overload - a situation in which a person is unintentionally flooded with unwelcome information. Players engage in two information activities to prevent this. The first of these is blunting - the direct rejection of unwelcome information (as per Baker, 1996). The second is information avoidance, the practice of performing one's searches and other types of information acquisition in a manner that makes it less likely that unwelcome information is encountered (Harviainen, Gough and Sköld, 2012). MMORPG players have been documented to extensively use information acquisition methods such as active scanning and non-directed monitoring, which are ways of being open to encountering new information (Adams, 2009). Yet they tend to utilize these only in situations where the risk of encountering spoilers is low (Harviainen and Savolainen, 2014).

This practice carries over into the culture of play, affecting the way in which information is shared and traded. Spoiler warnings exemplify this, as does avoidance. It is considered proper behavior not to offer information about play content without being asked for it first, but as the asking is sometimes stigmatizing, fewer information is traded than would be needed. As observed by Richard D. Gough (e.g., Harviainen, Gough and Sköld, 2012), players avoid information overload by as much as possible avoiding information sources that might cause it, even to the detriment of finding what they actually require. Formal organizations such as guilds and corporations can regulate these processes better, to some extent, through both their rules and the use of dedicated ICTs (see Warmelink, 2014).

Designers of a game design both the artifact as well as the emergent play processes that it can provide (Wardrip-Fruin, 2009). Players, however, are known to go against designer intent in many ways. Some of those ways are nevertheless beneficial to the game experience. Myers (2010) calls this "functional bad play", and it can be seen as an example of what, following Bartle (see e.g., 2014), is often considered "exploration" or "spading" of the game, including both its fictional environment and code (i.e., the IR core). Again, this follows Land's (1992, pp. 12-13) assessment of information systems in general, that even the most rigidly defined system will be used by its 
information users in ways which were neither planned for nor anticipated by its designers. The exploration, too, carries the risk of overload, spoilers and exploitable bugs that become too popular, for both the exploring players and to anyone with whom they share the information.

Therefore, we argue, some players - in especially organizations - are also contributing with silence, instead of just with sharing. The implicit trade rule can be described as "contribute as much as possible to the tasks at hand, but do not spoil anything for other members". Those who follow the rule well are attributed social capital and increasing guild privileges. Those who do not are stigmatized, either as freeloaders, or 'inconsiderate assholes'.

\section{DISCUSSION: BARTER - OR EMERGENT CURRENCY?}

In addition to information being useful and sometimes intrinsically valuable in itself, the practice of creating and sharing information goods functions as a form of payment in virtual worlds. In effect, information practices approach the state of becoming a type of service-ascurrency, in the sense of something bartered so often by all parties that it becomes a means of localized financial exchange (see e.g., Saito and Morino, 2010). Such an emergence tends to take place without any explicit contract by anyone, through popularity (Lehdonvirta and Castronova, 2014). It can also be the result of an intentional coupling between money and barter, the same way that e.g., a credit card company shares its member list with a retailer and gets the members a discount in turn from that retailer (see Normann, 2001, pp. 26-27). Players have already adopted as currencies some items, where a formal currency has been missing. For example, in the multiplayer combat game Team Fortress 2 players adopted keys as a currency, since they were freely tradable, their price was set and predictable, and all the keys had similar functional value.

Money traditionally has three key uses: as a means of exchange, as a store of value, and as a measure of value (see e.g., Jevons, 1875; Lehdonvirta and Castronova, 2014, pp. 177-179). Of these, information practices only really fulfill the first, because they are neither really fungible (of equal value between each other) nor stable or durable. Neither are or were, however, all forms of real-world currency: gold coins may be of differing purity, and just like information can become outdated or obsolete, cocoa beans spoil over time.

Information practices, as a kind of service-as-currency, have certain advantages as well. They are highly recognizable, extremely easy to transport and handle, flauntable, easy to verify, and occasionally also divisible (see Lehdonvirta and Castronova, 2014, pp. 179-184). The area within which they are accepted as payment is quite limited, however, and tends to extend only to the game itself and the forums (etc.) where it is described, plus occasionally to a larger gaming community, if the information practices are exchanged into cultural capital (see Harviainen and Savolainen, 2014). As barter processes are dependent on negotiation and network building, and value networks in turn usually on barter (Normann, 2001, p. 109), in theory nothing prevents information practices' value from spreading even further.

In especially some task-oriented guilds and EVE corporations, we can see the currency-forming development taking place, as information practices get traded for e.g. privileges and Dragon Kill Points, sometimes even at a regular rate (see Warmelink, 2014). Given the value of information as a good in virtual worlds, and the value given to information practices as services in play, we predict that it will spread in the future. Certain information practices seem to hold potential for becoming virtual currency in virtual worlds, yet remain so unstable that it is unlikely that their use in that form will spread beyond small communities like strongly task-oriented guilds.

\section{CONCLUSIONS}

In this article, we have shown how an information trade emerges from the systems that are inherent in an MMORPG. Players pay for services and items, and demonstrate group commitment, through the practices of seeking, creating, sharing and withholding information. As a result of the systems structure of a MMORPG, many information practices within the virtual world of the game, or outside it but related to play, become commodified. Players trade in that information, share it, and search for it, as a way to both garner cultural capital and to show commitment to their intra-game organizations.

In 'real world' economic conduct, more accurate information is always regarded as positive thing. Games, however, are interesting environments with respect to the amount of information players and organizations might want to have. Since the main activity in games is to seek information, which is done willingly by the players, having too much of information would render the game system useless. Therefore, even withholding information in these systems become valuable. This notion also pertains to other information practices, in situations where information is being consumed or created, and holds intrinsic experiential value, such as movies. In order to avoid sharing too much, players furthermore withhold information they consider unsuitable for trading or open provision. This way, they both shield other players from spoilers and make sure that the information they have stays valuable.

However, not all activities in games hold similarities to intrinsically motivated exploration of information. Therefore, players are sometimes eager to pay for information (services) with money they have earned somewhere else. Real money trade provides access to more content and thus more information. Some players see certain facets of it as a sidestepping, often an unfair one, of the way play is supposed 
(by at least them) to proceed. Roughly put, buying extra content with RMT is fine, buying extra powers ("paying to win") is not. The systems themselves, however, are often designed to support and even promote RMT, in order to create revenue for the publishers. As a result, the information economy of an MMORPG becomes splintered, with some seeing effort-requiring information practices as a sign of dedication, while others pay with real-world money to get access to content that is exclusive, to bypass game tasks that they do not want to complete, or to win a competitive situation by paying more than the opponent does.

Information practices in general, as a form of payment, do not fulfill all the criteria of a good currency, and it can thus be argued that they are just a target of barter rather than means of exchange. This is especially true since one could easily find much of the information online for free, so it is often in truth more a trade on effort and time rather than the information content itself. As this article shows, however, that "lousy" money has in virtual worlds reached a level of acceptance as a form of payment far stronger than similar practices hold in the real world. This is due to the fact that it controls access to content, and thus enjoyable play.

The results furthermore show that in order to understand the economy of an MMORPG, researchers have to look deeper into not just the trade of in-game items, virtual fiat money (such as in-game gold) and the real money trade, but also into services used as payment in those realms. While the same individual pieces of information may not exchange proverbial hands to constitute a currency, the practice itself of using information for trade, at least sometimes, seems to do so.

\section{REFERENCES}

Alha, K., Koskinen, E., Paavilainen, J., Hamari, J. and Kinnunen, J. (2014), ’Free-to-Play games: professionals' perspectives", in Proceedings of Nordic DiGRA 2014 (Gotland, Sweden, May 29, 2014).

Adams, S.S. (2009), "What games have to offer: information behavior and meaning-making in virtual play spaces", Librarv Trends, Vol. 57 No. 4, pp. 676-693.

Baker, L.M. (1996), “A study of the nature of information needed by women with multiple sclerosis”, Library \& Information Science Research, Vol. 18 No. 1, pp. 67-81.

Bartle, R.A. (2014), "Design principles: use and misuse”, in Quandt, T. and Kröger, S. (Eds.), Multiplayer: The Social Aspects of Digital Gaming, Routledge, New York, NY, pp. 10-22.

Bourdieu, P. (1984), Distinction: A Social Critique of the Judgement of Taste, Harvard University Press, Cambridge, MA.

Buckland, M. (1991), Information and Information Systems, Praeger, New York, NY.

Castronova, E. (2005), Synthetic Worlds: The Business and Culture of Online Games, University of Chicago Press, Chicago, IL.

Checkland, P. and Holwell, S. (1998) Information, Systems and Information Systems, John Wiley, New York, NY.

Chen, M.G. (2009), "Communication, coordination and camaraderie in World of Warcraft", Games and Culture, Vol. 4 No. 1, pp. $47-73$.

Consalvo, M. (2007), Cheating: Gaining Advantage in Videogames, MIT Press, Cambridge, MA.

Cosijn, E. and Ingwersen, P. (2000), "Dimensions of relevance”, Information Processing \& Management, Vol. 36, pp. 533-550.

Crookall. D., Oxford, R. and Saunders, D. (1987), "Towards a reconceptualization of simulation: from representation to reality", Simulation/Games for Learning, Vol. 17 No. 4, pp. 147-171.

EVE Online. CCP Games, 2003.

EverQuest, Sony Online Entertainment, 1999.

Galliers, R.D. (1992), “Choosing information systems research approaches”, in Galliers, R.D. (Ed.), Information Systems Research: Issues, Methods and Practical Guidelines, Blackwell Scientific, Oxford.

Hamari, J. and Lehdonvirta, V. (2010), "Game design as marketing: how game mechanics create demand for virtual goods", International Journal of Business Science \& Applied Management, Vol. 5 No. 1, pp. 14-29.

Hamari, J. (2011), "Perspectives from behavioral economics to analyzing game design patterns: loss aversion in social games", in Proceedings of CHI'2011, Social games workshop, (Vancouver, Canada, May 7-12, 2011).

Hamari, J. and Eranti, V. (2011), "Framework for designing and evaluating game achievements", in Proceedings of Digra 2011 Conference: Think Design Play, Hilversum, Netherlands, September 14-17, 2011.

Hamari, J. (2013), "Transforming Homo Economicus into Homo Ludens: a field experiment on gamification in a utilitarian peer-to-peer trading service", Electronic Commerce Research and Applications, Vol. 12 No. 4, pp. 236-245.

Hamari, J., Huotari, K. and Tolvanen, J. (2014 in press), "Gamification and economics", in Walz, S.P. and Deterding, S. (Eds.), The Gameful World: Approaches, Issues, Applications. The MIT Press, Cambridge, MA.

Hamari, J. (2015), Gamification: Motivations and Effects. Doctoral Dissertation, Aalto University.

Hansen, P. and Järvelin, K. (2005), “Collaborative information retrieval in an information-intensive domain”, Information Processing \& Management, Vol. 41, pp. 1101-1119.

Harviainen, J.T. (2012), "Ritualistic games, boundary control and information uncertainty", Simulation \& Gaming, Vol. 43 No. $4,506-527$. 
Harviainen, J.T., Gough, R.D., and Sköld, O. (2012), “Information behavior in game-related social media”, in Widén, G. and Holmberg, K. (Eds.), Social Information Research, Emerald, Bradford, pp. 149-171.

Harviainen, J.T. and Savolainen, R. (2014), "Information as capability for action and capital in synthetic worlds", Information Research, Vol. 19 No. 4, ISIC paper 12.

Ingwersen, P. and Järvelin, K. (2005), The Turn: Integration of Information Seeking and Retrieval in Context, Springer, Dordrecht. Jevons, W.S. (1875), Money and the Mechanism of Exchange, Appleton, New York, NY.

Jørgensen, K. (2013), Gameworld Interfaces, MIT Press, Cambridge, MA.

Kallio, K.P., Mäyrä, F. and Kaipainen, K. (2011), "At least nine ways to play: approaching gamer mentalities", Games and Culture, Vol. 6 No. 4, pp. 327-353.

Klabbers, J.H.G. (2009), The Magic Circle: Principles of Gaming \& Simulation, Sense Publishers, Rotterdam.

Koivisto, J. and Hamari, J. (2014), ”Demographic differences in perceived benefits from gamification”, Computers in Human Behavior, Vol. 35, pp. 179-188.

Land, F. (1987), “Social aspects of information systems”, in Piercy, N. (Ed.), Management Information Systems: The Technology Challenge, Croom Helm, London.

Land, F. (1992), “The information systems domain”, in Galliers, R.D. (Ed.), Information Systems Research: Issues, Methods and Practical Guidelines, Blackwell Scientific, Oxford.

Lastowka, F.G. and Hunter, D. (2004), "The laws of the virtual worlds", California Law Review, Vol. 92 No. 1, pp. 1-73.

Lehdonvirta, V. and Castronova, E. (2014), Virtual Economies: Design and Analvsis, MIT Press, Cambridge, MA.

Lehdonvirta, V., Wilska, T.-A. and Johnson, M. (2009), "Virtual consumerism: case Habbo Hotel", Information. Communication \& Societv, Vol. 12 No. 7, pp. 1059-1079.

Lin, H. and Sun, C.T. (2011), "Cash trade in free-to-play online games", Games and Culture, Vol. 6 No. 3, pp. $270-287$.

Malone, K.L. (2009), "Dragon kill points: the economics of power gamers”, Games and Culture Vol. 4 No. 3, pp. 296-316.

McKenzie, P.J. (2003), "A model of information practices in accounts of everyday-life information seeking”, Journal of Documentation, Vol. 59 No. 1, pp. 19-40.

Mintzberg, H. (1983), Structure in Fives: Designing Effective Organizations, Prentice-Hall, Englewood Cliffs, NJ.

Mon, L.M. (2012), "Professional avatars: librarians and educators in virtual worlds", Journal of Documentation, Vol. 68 No. 3, pp. 318329.

Myers, D. (2010), Plav Redux: The Form of Computer Games, University of Michigan Press, Ann Arbor, MI.

Normann, R. (2001). Reframing Business: When the Map Changes the Landscape. New York: Wiley.

Paavilainen, J., Hamari, J., Stenros, J. and Kinnunen, J. (2013), ”Social network games: players' perspectives”, Simulation \& Gaming, Vol. 44 No. 6, pp. 794-820.

Saito, K. and Morino, E. (2010), "The brighter side of risks in peer-to-peer barter relationships". Future Generation Computer Svstems, Vol. 26 No. 8, pp. 1300-1316.

Saracevic, T. (1996), "Relevance reconsidered '96", in Ingwersen, P. and Pors, N.O. (Eds.), Information Science: Integration in Perspective, Royal School of Library and Information Science, Copenhagen.

Savolainen, R. (1995), "Everyday life information seeking: approaching information seeking in the context of "way of life"”, Librarv \& Information Science Research, Vol. 17 No. 3, pp. 259-294.

Second Life. Linden Research, 2003.

Shah, C. (2012), Collaborative Information Seeking: The Art and Science of Making the Whole Greater than the Sum of All, Springer, Dordrecht.

Shapiro, C. and Varian, H.R. (1999), Information Rules: A Strategic Guide to the Network Economy, Harvard Business School Press, Boston, MA.

Shaw, W. and Culkin, P.B. (1987), "Systems that inform: emerging trends in library automation and network development", Annual Review of Information Science and Technologv, Vol. 22, pp. 265-292.

Silverman, M. and Simon, B. (2009), "Discipline and dragon kill points in the online power game", Games and Culture, Vol. 4 No. 4 , pp. 353-378

Sköld, O., Adams, S., Harviainen, J.T. and Huvila, I. (in press), “Studying games from the viewpoint of information”, in Lankoski, P. and Björk, S. (Eds.), Game Research Methods, ETC Press.

Stenros, J., Paavilainen, J. and Mäyrä, F. (2011), "Social interaction in games”, International Journal of Arts and Technologv, Vol. 4 No. 3 , pp. 342-358.

Team Fortress 2, Valve, 2007.

Ultima Online, Origin Systems, 1997.

Vakkari P. (1999), "Task complexity, problem structure and information actions: integrating studies on information seeking and retrieval", Information Processing \& Management, Vol. 35, pp. 819-837. 
Vesa, M. (2013), There be Dragons: An Ethnographic Inquiry into the Strategic Practices and Process of World of Warcraft Gaming Groups, Doctoral Dissertation, Hanken School of Economics.

Wardrip-Fruin, N. (2009), Expressive Processing: Digital Fictions, Computer Games, and Software Studies, MIT Press, Cambridge, MA. Warmelink, H. (2014), Online Gaming and Playful Organization, Routledge, New York, NY.

Williams, D., Ducheneaut, N., Xiong, L., Zhang, Y., Yee, N. and Nickell, E. (2006), "From tree house to barracks: the social life of guilds in World of Warcraft", Games and Culture, Vol. 1 No. 4, pp. 338-361.

World of Warcraft. Blizzard Entertainment, 2004.

\section{Acknowledgments (if applicable):}

The authors wish to thank Janne Paavilainen and Reijo Savolainen for their helpful comments during the preparation of this article. This research has been partially supported by an individual study grant from the Finnish Cultural Foundation as well as partially carried out as part of research projects $(40311 / 12,40134 / 13)$ funded by the Finnish Funding Agency for Technology and Innovation (TEKES). 